\title{
Preparation \& Characterization of Some Poly Ester-Amide as Drug Polymers
}

\author{
Abeer Abdul RazakMohammed ${ }^{1}$; ManalAzat Aziz ${ }^{2}$ \\ ${ }^{1}$ University of Mustanseriya, College of Science, Department of Chemistry; \\ ${ }^{2}$ Al-Mansour Institution of Medical Technology Middle Technical University
}

\begin{abstract}
In this research,lactide was synthesized by ring opening polymerization then reacted with amino acid to obtain drug polymer,poly (ester-amide)by condensation polymerization at $90 \mathrm{C}^{\circ}$ with mixture DMF\&dioxane(1:1), then the biodegradable polymers substituted by some hydroxyl drug units such as 3-hydroxy pyridine. The action of polymeric drugs depends on hydrolytic \& cleavage of the drug moiety from the polymer,this give advantage of late $\&$ sustained release of drug over long time with decrease of side effects. The new polymers wich has bioactive group's moiety were measured physical properties and characterized by FTIR \& UV spectroscopy and TG thermogram.
\end{abstract}

Keywords:- lactide ; aminoacid ; biodegradable ; polymeric drugs

\section{Introduction}

Human health is threatened by autoimmune,neurodegenerative,metabolic and cancer disease, just to mention a few, which are difficult to treat with systemically, delivered drugs ${ }^{(1)}$.Conventional pharmacotherapy involves the use of drugs whose absorption and therefore bioavailability dependes on many factors, such as solubility, molecular weight, number of bonds per hydrogen atom of the molecule ,and chemical stability, all of wich can hinder the achievement of therapeutic response ${ }^{(2,3)}$.

Research is being conducted into new formulations that ensure a greater pharmacological response, which in turn would lead to lower doses and therefore the minimization of side effects .Thus, it is necessary to improve the bioavailability of drugs .Bioavailability is affected by several factors ,including the physical and chemical characteristics of the drug ${ }^{(4)}$,the dose and concentration, the frequency of dosing, and the administration route.

Therefore ,research into drug delivery systems seeks to improve the pharmacological activityof drugs by enhancing pharmacokinetics (absorption, distribution,metabolism and excretion) and also by amending pharmacodynamics ptoperties, such as the mechanism of action, pharmacological response, and affinity to the site of action ${ }^{(5)}$

Hence, the main function of polymeric carriers is to transport drugs to the site of action .Drugs are protected from interacting with other molecules which could caise a change in the chemical structure of the active ingredient causing it to loose its pharmaceutical action ${ }^{(6,7)}$.Moreover, polymeric carriers avoid the intreraction of the drug with macromolecules

In the case of biodegradable polymers, another option to consider in their design is the chemical structure of the polymer (degree of hydrophobicity , covalent bonds between monomers ,etc.), sincs the speed and degradation condition, and therefore, the rate and site of drug release,can be modulated depending on the chemical structure of the polymer used ${ }^{(8)}$.

If the polymer is not biodegradable, the drug can be covalently attached to the polymeric structure by a linker which can be degrade $\mathrm{d}$ under different conditions such as in an acidic medium or by different enzymes ${ }^{(9)}$.On the other hand, targets can be bound covalently to the surface which will help the directionality of the vector to the site of action. 
Lactide is iactone cyclic di-ester.,Tow molecules of lactic acid can be dehydrated to lactide, a cyclic lactone avarity of catalysts can polymerize lactide to either ${ }^{(10)}$ heterocic or syndiotacticpolylactide ${ }^{(11)}$.

Lactide is one of the most promising biodegradable polymers (biopolymers).The basic aim of prodrug designs to eliminate undesirable drug properties,such as low solubility in water or lipid membrane,low target selectivity,chemical instability ${ }^{(12)}$, undesirable taste,irraitation or pain after local administration,pre systemic metabolism and toxicity .

\section{Materials \& Methods}

Glycine,arginine,3-Hydroxy

pyridine ,ether,zincpowder, $\mathrm{NaOH}$,dioxane, and lactic acid were purchased fromAldric,Dimethylformamide was purchased from Merck.

FTIR spectra were reached by $(4000-900) \mathrm{cm}^{-1}$ on shimatau spectrometer using $\mathrm{KBr}$ pellet .

Ultra violet spectra were recorded using Shimadzu (UV-Vis)-160, TGA and DTG were performed using CINTRAS-UV(13).

\section{Synthesis of L-lactide (P1):-}

The synthesis of l-lactide was carried out according to the procedure that was reported by Bendix with some modification including the use of $\mathrm{Zn}$ as catalyst.

Lactic acid (88gm,0.97mole)and zinc powder (0.44gm,0.0146mol.) were added to $100 \mathrm{ml}$ round bottom flask containing magnetic stirrer and the mixture was stirred for 15 minutes, then the reaction was placed in a sand bath at $130 \mathrm{C}^{\circ}$ and the temperature raised to about $200 \mathrm{C}^{\circ}$ with continuous stirring under vacuum pressure for four hr.Theflsk content was left to cool down to room temperature with a continuous stirring until colorless viscous material was formed,chloroform (100ml)was added to the reaction flask, and then the content of the flask transferred to a separate funnel,washed by $(45 \mathrm{ml})$ of $\mathrm{NaOHsolution.The} \mathrm{organic} \mathrm{layer} \mathrm{was} \mathrm{received} \mathrm{and}$ the solvent removed under reduce pressure ${ }^{(14,15)}$,a light yellow viscous was collected with yield $70 \%$ and softening point $270 \mathrm{C}^{\circ}$.

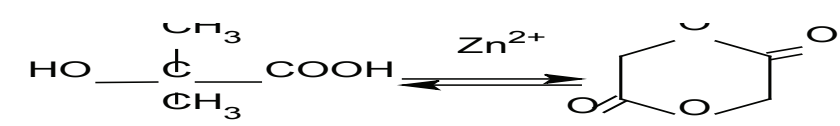

\section{Poly condensation of some amino acids with poly lactide(P2,P3) :-}

(1 mole,1.19gm) of prepared lactide was dissolved in $(5 \mathrm{ml})$ mixture of dioxane and DMF (1mole,2gm)of amino acid such as glycine was added, the mixture was heated to $90 \mathrm{C}^{\circ}$ for $1 \mathrm{hr}$. The brown product was washed with ether and was dried at $50 \mathrm{C}^{\circ}$ in vacuum oven, rigid product .

Modification of prepared polymers with heterocyclic compound(P4,P5).

(1.7gm,0.014mole)of 3-hydroxy pyridine was dissolved in (1ml)DMF and (10ml)dioxane,the (2gm,0.014mole)of preparing polymers (P2,P3) which dissolved in (1ml)DMF and (10ml)dioxanewas added with stirring in flask equipped with condenser, then reflexed $3 \mathrm{hr}$.The solvent was evaporated under vacuum, the substituted polymers were washed by ether for several times and dried,physical properties .

\section{Finding}

Recently ,studies on biodegradable polymers has increased in importance due to wide range of biomedicine applications an addition to packaging and agriculture applications .(lactide importance) in the present study lactate polymers which have biological activities was prepared studied.

FTIR spectrum of lactide( $\mathrm{P} 1)$ shows the absorption of $(\mathrm{C}=\mathrm{O})$ ester in $(1724) \mathrm{cm}^{-1}$, the absorption of $(\mathrm{C}-\mathrm{O})$ was appeared at $(1207) \mathrm{cm}^{-1}$,finally, the absorption of (C-H)aliphatic was appeared at $(2943) \mathrm{cm}^{-1},(2887) \mathrm{cm}^{-1}$, figure 1.

FTIR specrum of (P4) shows absorption of bands at (3377) $\mathrm{cm}^{-1}$ due to $(\mathrm{OH})$ group of remaining carboxylic acid ,(1732) $\mathrm{cm}^{-1}$ due to $(\mathrm{C}=\mathrm{O})$ este,,$(1300) \mathrm{cm}^{-1}(\mathrm{C}-\mathrm{O})$ estere, $(1651) \mathrm{cm}^{-1}$ due to $(\mathrm{C}=\mathrm{O})$ amide,$(3522) \mathrm{cm}^{-1}$ due to $(\mathrm{NH})$ and at last, figure shows absorption bnnds of $(\mathrm{C}-\mathrm{H})$ aliphatic at (2026) $\mathrm{cm}^{-1},(2856) \mathrm{cm}^{-1}$ figure2.

FTIR specrum of (P5)shows absorption bands at (3308) $\mathrm{cm}^{-1}$ due to $(\mathrm{OH})$ group of remaining carboxylic acid ,(1734) $\mathrm{cm}^{-1}$ due to $(\mathrm{C}=\mathrm{O})$ ester , (1307) $\mathrm{cm}^{-1}$ due to 
(C-O)ester,,$(1647) \mathrm{cm}^{-1}$ due to $(\mathrm{C}=\mathrm{O})$ amide , $(3290) \mathrm{cm}^{-1}$ due to $(\mathrm{NH})$ and at last ,we can see also absorption bands at (2928),(2870) $\mathrm{cm}^{-1}$ due to $(\mathrm{C}-\mathrm{H})$ aliphatic, figure3.

ultraviolet spectrum of (P5) shows an absorption band at (280)nm,figure4.

The thermal stability of the prepared polymer(P1) was investigated by thermogravimetric analysis (TGA).This technique is based on measuring the weight loss as a function of time at constant temperature and a function of tempreture at constant rate of heating. In the present study, the thermal stability of the prepared polymer was tested by measuring the sample weight as a function of temperature Figure5. The thermal measurement gave valuable information about thermal stability of the prepared compound in four steps of weight loss-temperature .This high thermal resistance indicated the high molecular weight of the prepared polymers with high interaction between hydrogen bonding through the polymer chain.

The weight loss of P2,P3 were measured as a function of temperature which gave valuable information about the thermal stability of the P2,P3 polymers as shown in, figure6.

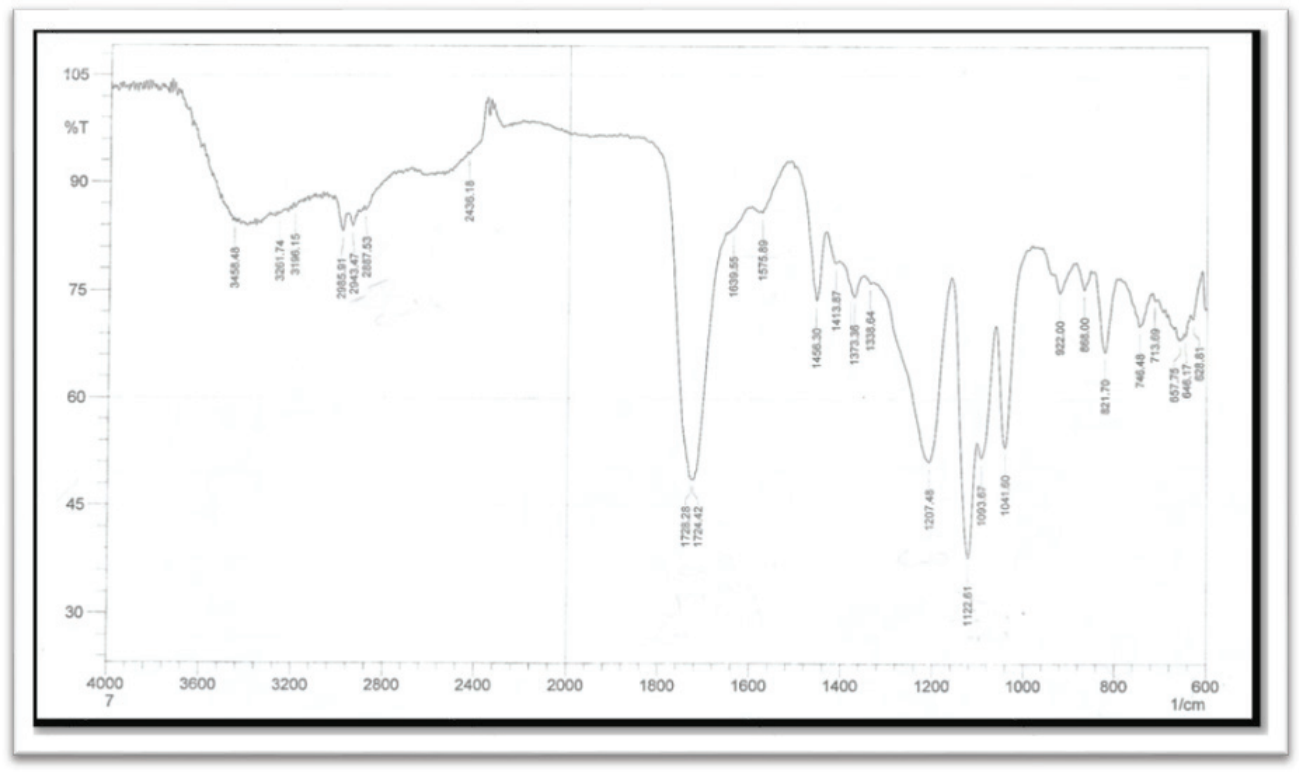

Figure(1) : FTIR spectrum of P1

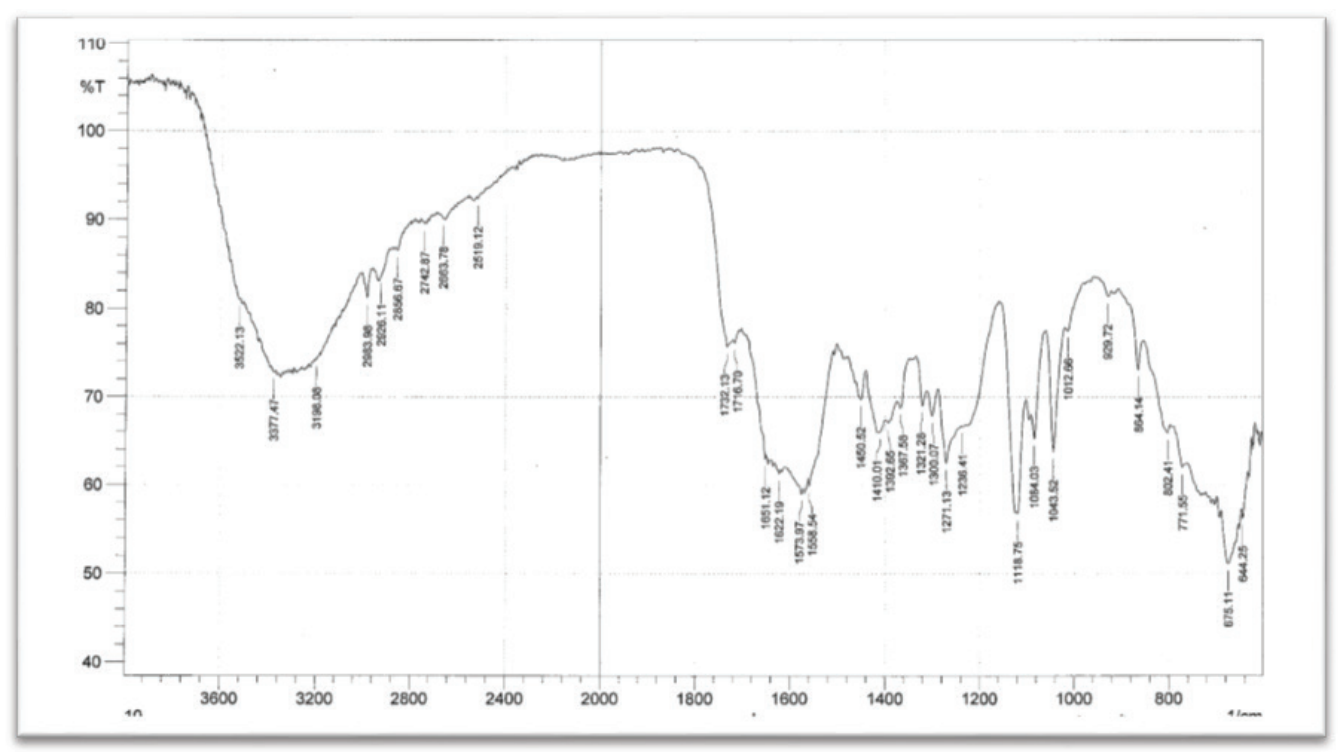

Figure (2) : FTIR spectrum of (P4) 


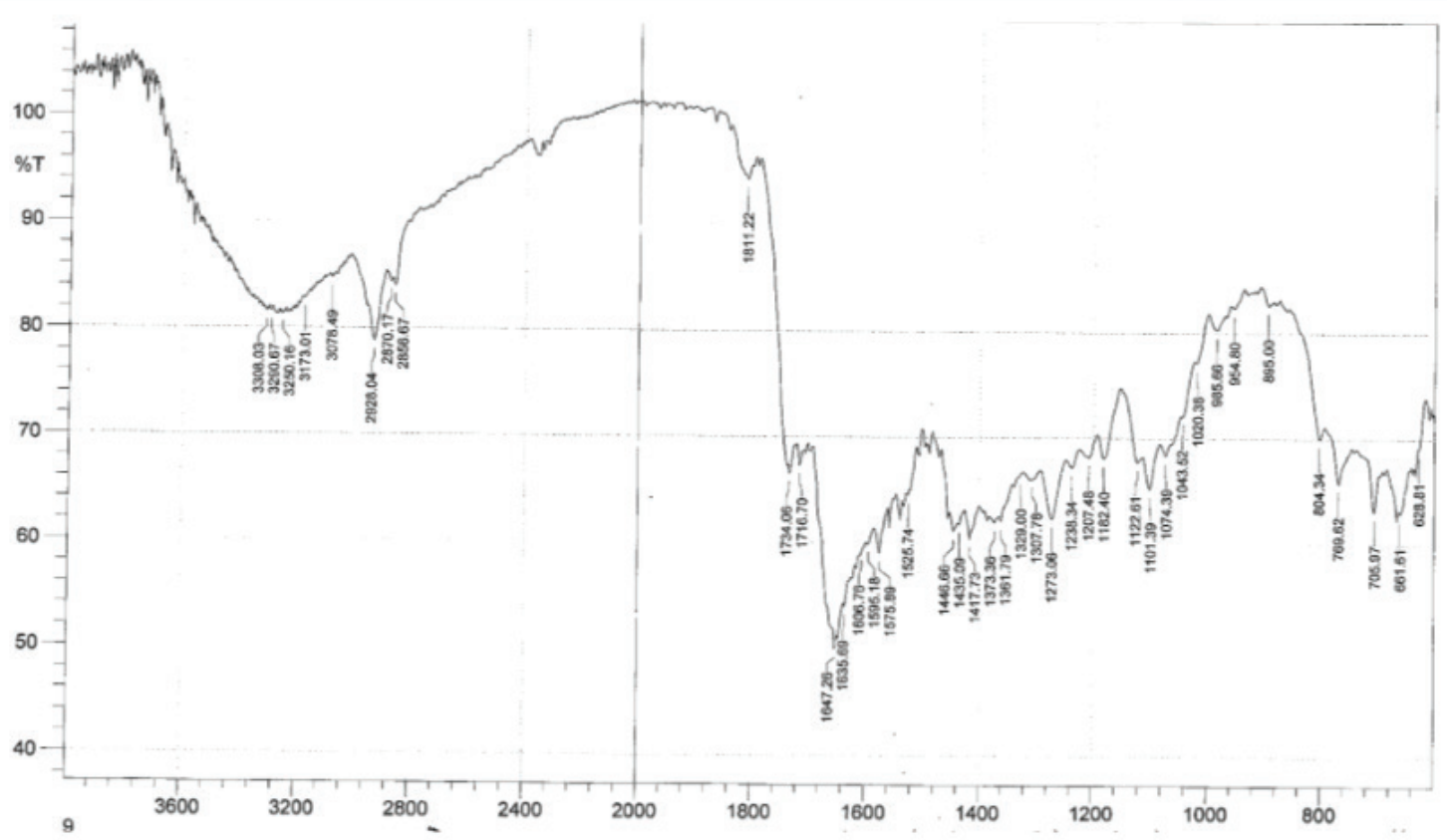

Figure (3) : FTIR spectrum of (P5)

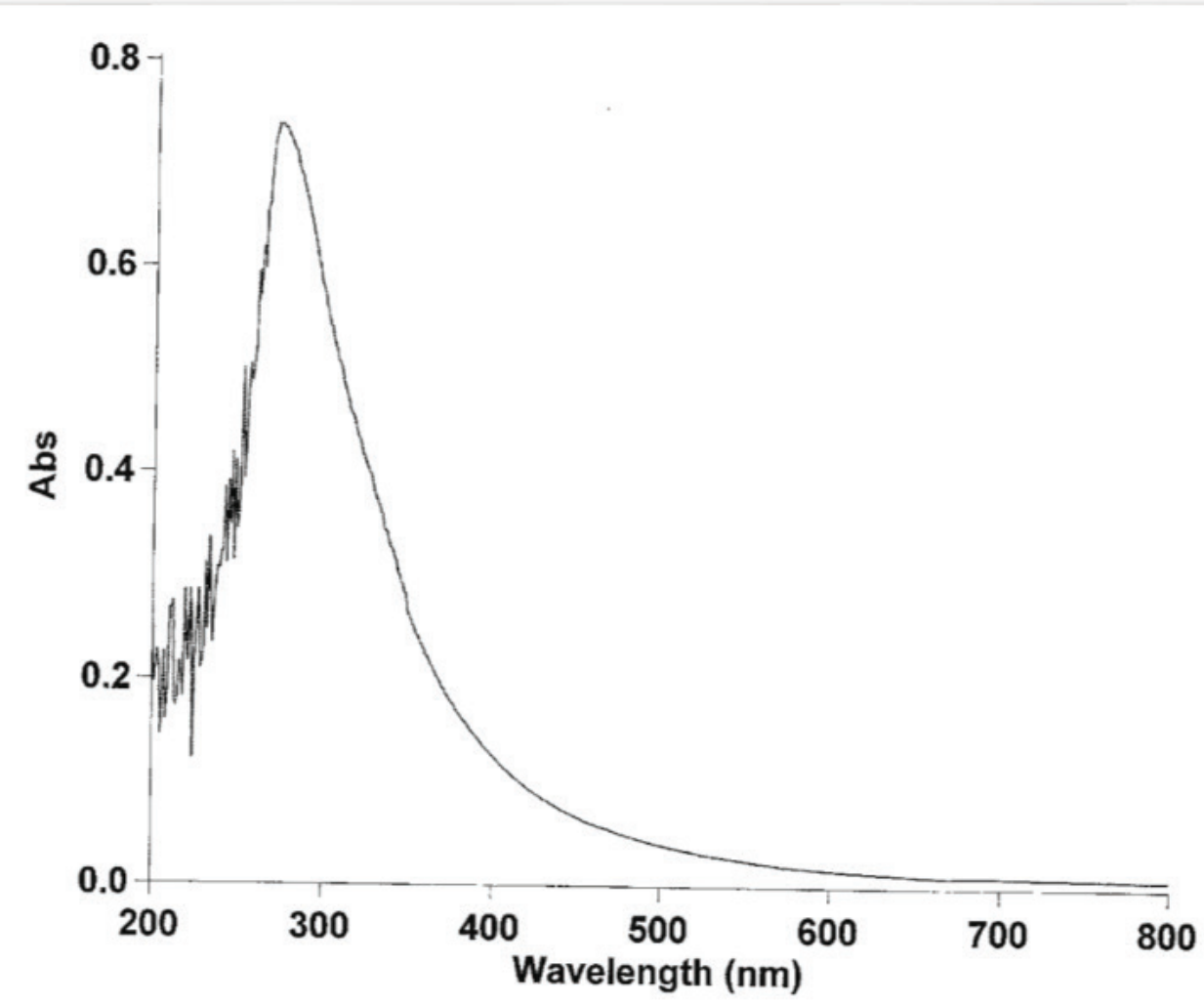

Figure(4): Ultraviolet spectrum of P5 


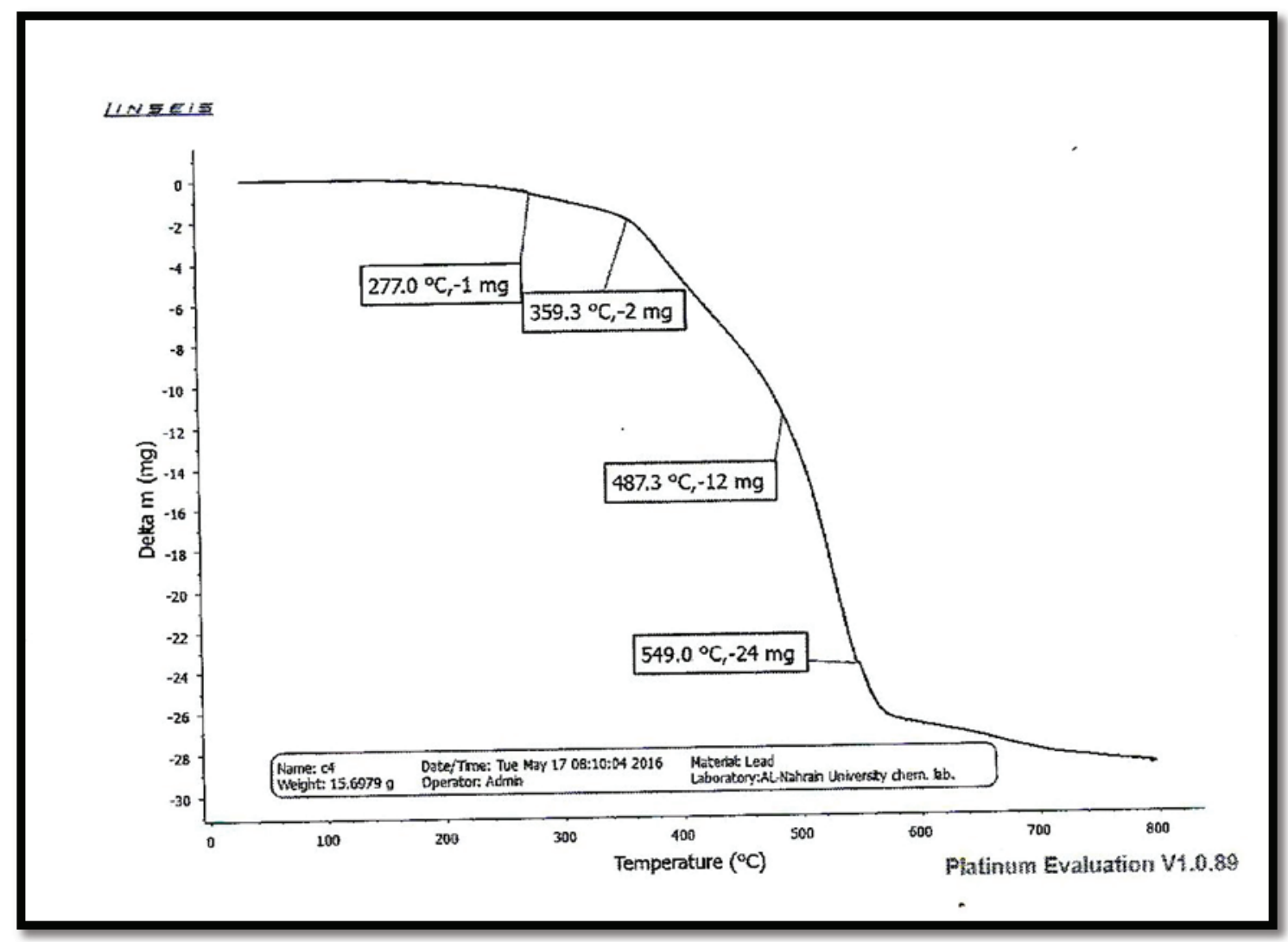

Figure (5) : thermal stability measurement of P1

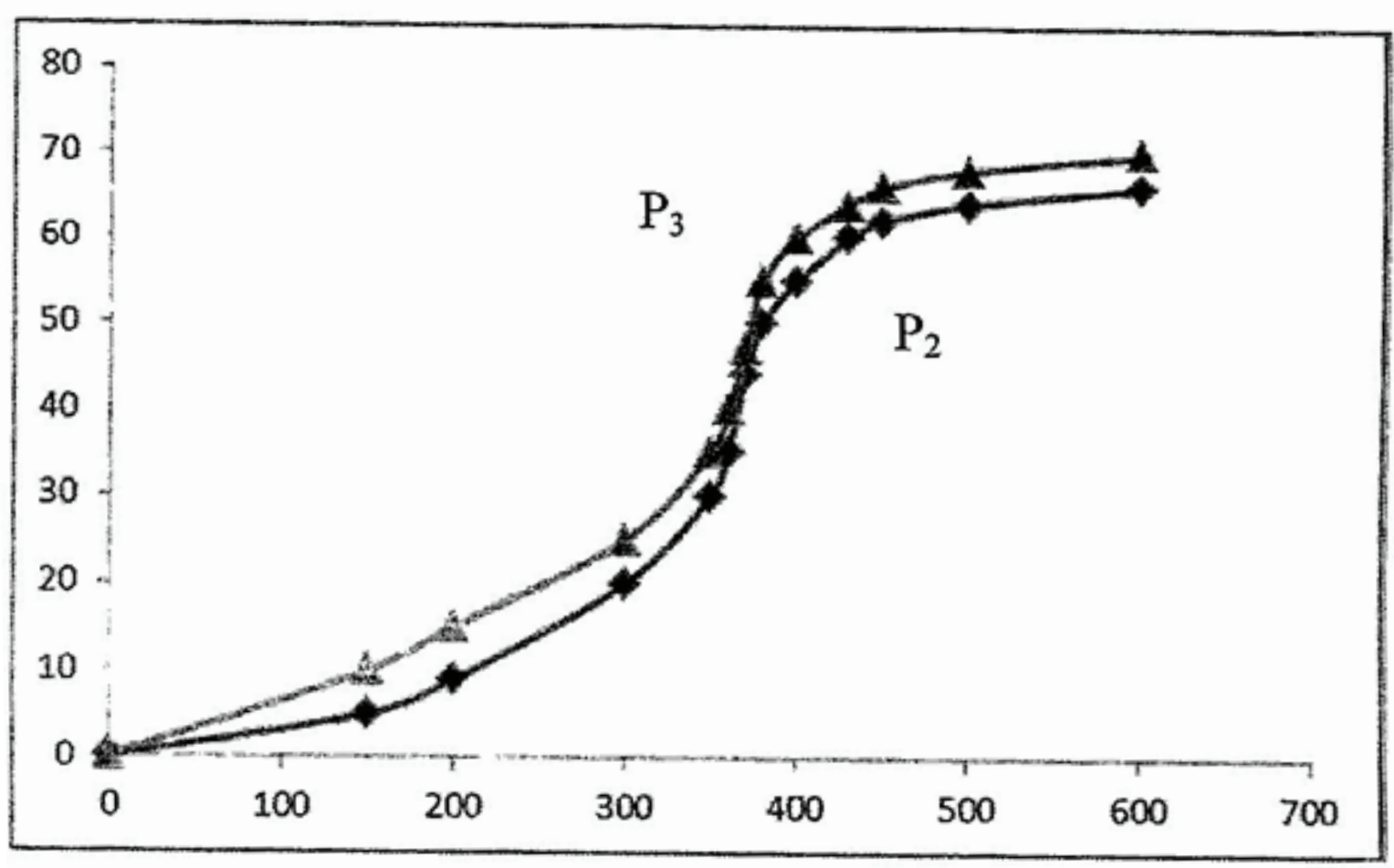

Figure (6) : Thermal stability measurement of P2,P3 
Conflict of Interest : None

Source of Funding : Self

Ethical Clearance : From my college .

\section{Reference}

1- Schierholz,J.M.Rump.A.;Pulverer,G.Drug. Res.1997.

2- Maniar,M;Domp,A;Haffer,A;Shah;Drug Delivery J.J.Control Release1994,30,233-239.

3- Wang,B,Siahaan,T.Sohero,R.A.In:Drug Delivery :principles and applications ,Ed.Wiley ,Inc,2005.

4- McGee,J,P.;Davis ，S.S.;O,Hagan,D.T.J.Control Release994,31,55-60.

5- Michael E.A .In :Translation of Pharmaceutics .The Science of Dosage Form Design,2e,2002.

6- Nowotnik,D.P,;Cvitkovic ,E.Adv.Drug Deliv. Rev.2009,61,1214-1219.

7- Wedge,S.R.; Duncan, R; Kopeckova,P. Br.J.Cancer.,1991,63,546-549.
8- DUCAN,B.Nat.Rev.Drug Discov.2003,2,347-360.

9- Verma,R.K.;Mishra,B,Garg S.Drug Deev.Ind. Pharma.2000,26,695-708.

10- Albertsson ,A-Ch.AndSrivastava,R.K.(2008). Recent development in enzyme-catalyzed ring opening polz.Advanced Drug Delivery Reviews ,Vol.60,No.9,(June2008),pp.1077-ISSNO169409x.

11- Rokicki,R.(2000).Alipha cyclic carbonate and spiroothocarbonates as monomers .Progress in polymer science, Vol .25, (January 2000),pp.259342,ISSN0079-6700.

12- L.B.Goldden ,J.physiol.588(2008).

13- Xinxin Feng,Literture Seminar ,Polymeric Conjugates for Anti -cancer Drug Delivery Septemper 16,(2010).

14- H.Ismaail,Z.Ahmed and F.W.Yew,Journal of physical science ,22(2)P.51-63(2011).

15- Eedener K.,Dusun S.,Turk.J.Chem.,26 (p.863-875),(2002). 